\title{
A Hybrid System for the Semantic Annotation of Sulco-Gyral Anatomy in MRI Images
}

\author{
Ammar Mechouche ${ }^{1}$, Xavier Morandi ${ }^{1,2}$, Christine Golbreich ${ }^{3,4}$, \\ and Bernard Gibaud ${ }^{1}$ \\ ${ }^{1}$ Unit/Project VisAGeS U746, INSERM - INRIA - CNRS - Univ-Rennes 1, France \\ \{Ammar.Mechouche, Bernard.Gibaud\}@irisa.fr \\ http://www.irisa.fr/visages/ \\ ${ }^{2}$ University Hospital of Rennes, Department of Neurosurgery, France \\ Xavier.Morandi@chu-rennes.fr \\ ${ }^{3}$ University of Versailles, Versailles \\ Christine.Golbreich@uvsq.fr \\ ${ }^{4}$ LIRMM UMR 5506, Montpellier, France \\ http://www.lirmm.fr/tatoo/
}

\begin{abstract}
This paper presents an interactive system for the annotation of brain anatomical structures in Magnetic Resonance Images. The system is based on hybrid knowledge and techniques. First, it exploits both numerical knowledge from atlases and symbolic knowledge from a ruleextended ontology represented in OWL, the Web ontology language, and combines them with graphical data about cortical sulci, automatically extracted from the images. Second, the annotations of the parts of gyri and of sulci located in a region of interest are obtained with different reasoning techniques: Constraint Satisfaction Solving and Description Logics techniques. Preliminary experiments have been achieved on normal and also pathological data. The results obtained so far are very promising.
\end{abstract}

\section{Introduction}

Semantic annotation associates meaningful labels to images, in order to highlight their information content. Semantic annotation is becoming a major issue regarding wide scale sharing of information on the web and is of critical importance in biomedical research, notably for translational research aiming at facilitating the exploitation of experimental data across several disciplines and scales [1. This is however a very challenging topic, due to the difficulty of defining consensual reference knowledge models, on the one hand, and to use them in image annotation tools, on the other hand. Such models, usually called ontologies, are often classified into two categories referred as lightweight and heavyweight ontologies, respectively [2] 1) simple term lists, thesauri or taxonomies, 2) highly expressive knowledge models, according to which instances are created, involving complex assertions and constraints. Our primary application field is the preparation of surgical procedures in neurosurgery. Actually, the precise identification of gyri and sulci around the lesional area is of primary importance because they provide

D. Metaxas et al. (Eds.): MICCAI 2008, Part I, LNCS 5241, pp. 807-814, 2008

(C) Springer-Verlag Berlin Heidelberg 2008 
useful landmarks for the surgeon during surgery, especially in eloquent cortex [3]. The annotation of images, in our case anatomical MRI images, has two objectives: 1) to help to identify precisely the gyri and sulci around the lesion, and to provide the detailed annotation data relating anatomical conceptual entities to graphical primitives extracted from the images; 2 ) to use these metadata for cases retrieval in an images database. Our approach significantly differs from classical approaches of parcellation of cortex in MRI images 445|6|7. Those are generally global (i.e. they analyze the whole cortex), have limited precision (i.e. they usually address gross anatomy), refer to simple term lists, are entirely automatic, mostly based on numerical priors (i.e. atlases), and primarily suited to normal anatomy. In contrast, our approach is local (i.e. focuses on a particular region in the image), may provide labels with high anatomical precision, can involve a human user participation, is based on symbolic prior knowledge (provided by a highly expressive ontology), and may be relevant to interpret pathological images as well as normal cases. The work presented here concerns a hybrid system designed according to the previous approach, i.e. a semantic approach, rather than morphometric or statistical. The knowledge supporting the labelling process consists of the mereo-topological relations between the different cortical features. This knowledge is described in an ontology of cortical gyri and sulci represented in OWL DI 1 , according to the description logics (DL) paradigm [8. The whole reasoning aims at producing instances of the ontology concerning the parts of sulci and of gyri involved in a set of graphical primitives extracted from the images (segments of cortical sulci, and parts of gyri called "patches"), that satisfy the axioms and constraints defined in the ontology. The very large number of possible combinations led us to adopt a hybrid approach, consisting in selecting a reasonable number of hypotheses for the labelling of patches, based on an atlas, and to select the valid combinations of such hypotheses, based on existing prior knowledge about the spatial arrangement of the gyri. This part constitutes a major extension of the method presented in 14. Section 2 provides an overview of the method and further details of the system components; section 3 describes our first experiments with both normal and pathological data; section 4 discusses these preliminary results and highlights the capabilities and current limitations of the system.

\section{Method}

\subsection{Definitions}

Definition 1: A segment (figure 1) is a part of an external trace of a sulcus. The segments are organized in a graph describing the connections between them.

Definition 2: A conventional separation (figure 1) is a fictitious line added by the user in order to connect two segments separated by a gyrus.

${ }^{1}$ Ontology Web Language based on Description Logic formalism http://www.w3.org/TR/owl-guide/ 

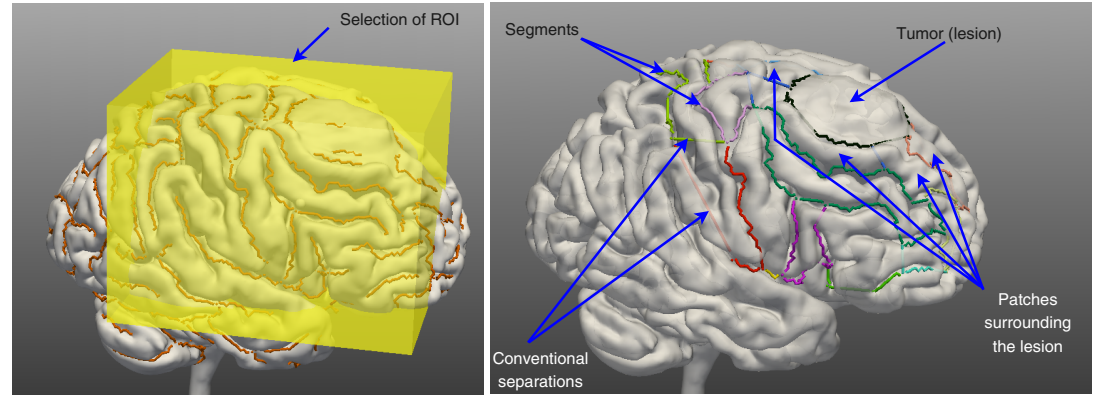

Fig. 1. ROI selection and Patches definition

Definition 3: A patch (figure 1) is a subset of the brain surface, corresponding to a part of a gyrus, and delimited by a set of continuous segments and conventional separations.

Definition 4: An interpretation consists of a set of labels associated with the patches and segments of a Region Of Interest (ROI); each patch and each segment has one label only.

Definition 5: A consistent interpretation is an interpretation, where each patch label and each segment label is consistent with our prior knowledge about the sulco-gyral anatomy and with the information supplied by the user.

\subsection{System Overview}

The overall labelling process involves three steps (figure 21): (1) the brain is segmented, the external traces of the sulci are extracted [9], the ROI is selected by the user, and the patches are defined (section 2.3); (2) patches identification: this is done by using Constraint Satisfaction Problem techniques (CSP) allowing to infer all the consistent interpretations for the patches with respect to prior knowledge about the spatial arrangement of the gyri (section 2.4). For that, the system uses a set of hypotheses (possible labels) derived from the matching of patch information with an atlas (SPAMs [10]); (3) sulci identification is done using the best interpretation computed for the patches and the logical definitions of the sulci in the ontology (section 2.5). The final annotations for the patches and the segments are generated in standard web languages to facilitate their exploitation by semantic web technologies. The paper focuses on (2) and (3).

\subsection{Delimitation and Extraction of the Patches}

The brain is automatically segmented from a T1-MRI scan and the graph of the external traces of the sulci is automatically extracted. Then, the user selects a ROI and the corresponding sub-graph is automatically extracted as shown on figure 1. Next, the user introduces a number of conventional separations, in order to produce a partition of the selected cortex area into a set of contiguous 


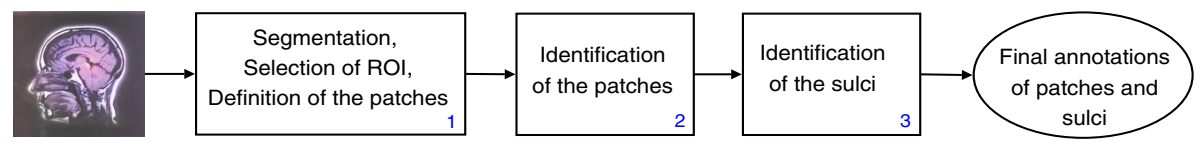

Fig. 2. General view of the labelling system

patches. Finally, the system computes the description of the topological relations and orientations between neighboring patches, and between the patches and the segments which form them. This description is represented in OWL (file1).

\subsection{Identification of the Patches}

Generation of Patches Labelling Hypotheses. The segments delimiting the patches within the ROI are realigned into the stereotaxic space (SPAMs space) thanks to the registration matrix produced during the segmentation process (figure 3). The position of each segment with respect to each SPAM is analyzed in order to determine whether it bounds this SPAM or not, and with which orientation. This analysis also provides a confidence index. All this information is represented in OWL (file2). The matching of information from file1 and file2 in the case of normal subjects is done by rules of the following form: Bounds $(x$, $y) \wedge \operatorname{SulcusPart}(x) \wedge \operatorname{Patch}(y) \wedge \operatorname{anteriorTo}(x, y) \wedge \operatorname{Bounds}(x, z) \wedge \operatorname{Gyrus}(z)$ $\wedge$ anteriorTo $(x, z) \rightarrow \operatorname{part} O f(y, z)$. This rule, for example, expresses that if we have a part of sulcus which bounds a given patch with an anterior orientation, and at the same time this part of sulcus bounds a given SPAM with the same orientation, then this patch is inferred to be part of the gyrus corresponding to this SPAM in the ontology. Six matching rules of this kind, corresponding to the six spatial orientations, are defined. Slightly different rules are used in pathological cases, taking into account only adjacency between the parts of sulci and the SPAMs, not orientations, because they might lead to erroneous decisions due to displacements related to the pathology. These rules assign to each patch a set of hypotheses. The correct label is assumed to belong to this set.

Determining the Consistent Interpretations Using a CSP Solver. A Constraint Satisfaction Problem (CSP) consists of a number of variables and a number of constraints. A variable is defined by its domain, i.e. the set of values that can be assigned to this variable. A constraint relates several variables and restricts the involved variables values to legal assignments. Constraint reasoning is the process of computing a solution to the given CSP, i.e. an assignment of values to the variables that satisfy all the given constraints on the variables [11. In our case (figure 3 -a), the patches represent the variables, the hypotheses computed for the patches represent the domains of the variables, and the spatial relations between the patches represent the constraints. The solutions of the CSP problem provide all possible interpretations for the patches with respect to our prior knowledge on the spatial arrangement of the gyri and parts of gyri. In order to find the best consistent interpretation for the patches among those computed by the CSP solver, the system 


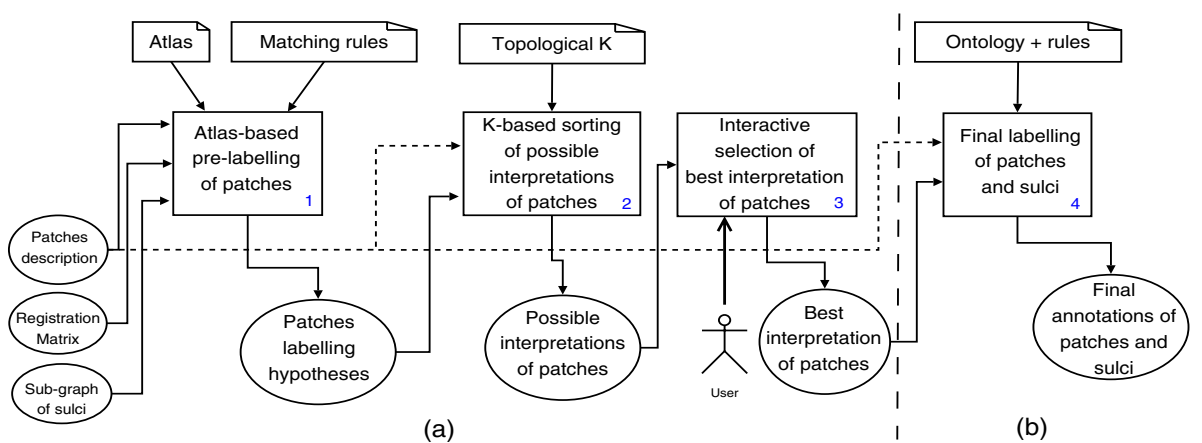

Fig. 3. The complete labelling process

allows interactions with the user. The latter is invited to select a preferred label for patches exhibiting different labels in different interpretations. Then the system eliminates those interpretations that are not consistent with the user information. The interactions are repeated until only one interpretation remains for the patches.

\subsection{Identification of the Segments}

To label the sulci segments the system uses the patches description, the best interpretation of the patches and the logical definitions of the sulci in the ontology (figure 3-b). The ontology contains the mereo-topological knowledge about the sulci and the gyri. To model the knowledge about the brain we have mainly used the Ono atlas [12, the Foundational Model of Anatomy (FMA2), and the expertise of a neuroanatomist. The concepts describing the sulci and gyri and their relations are defined in an ontology in OWL DL. An example of a concept definition of the ontology is the following: RightCentralSulcusPart $\equiv$ ( $\exists$ Bounds ( $\exists$ hasEntity $(\exists$ partOf RightPostCentralGyrus $)) \sqcap(\exists$ hasOrientation Anterior $))) \sqcap(\exists$ Bounds $((\exists$ hasEntity $(\exists$ partOf RightPreCentralGyrus $)) \sqcap$ ( $\exists$ hasOrientation Posterior $))) \sqcap(\forall$ Bounds $((\exists$ hasEntity $(\exists$ partOf RightPreCentralGyrus $)) \sqcup$ ( $\exists$ hasEntity ( $\exists$ partOf RightPostCentralGyrus))). This logical expression expresses that a part of the central sulcus of the right hemisphere bounds at least one part of the right postcentral gyrus with an anterior orientation and one part of the precentral gyrus with a posterior orientation, and only parts of such gyri. Our brain ontology contains for each hemisphere logical definitions of 49 gyri, 5 lobes, 3 operculum, 17 gyri parts, 44 sulci, 44 sulci parts (segments), and 31 relations. The ontology is enriched by some rules increasing its expressivity (see [14 for more details).

\section{Results}

\subsection{Material}

Preliminary experiments were made using T1-MRI images obtained with a $3 \mathrm{~T}$ scanner (Philips Achieva) from three normal subjects and two patients

\footnotetext{
2 http://sig.biostr.washington.edu/projects/fm/AboutFM.html
} 
(pathological subjects). In the two pathological cases the lesion was located in the right frontal lobe. The brain segmentation and the extraction of the external traces of the sulci were done with Brainvisa 3 tools and Vista 4 respectively. We have used 44 SPAMs corresponding to the gyri. The system is implemented in $\mathrm{C}++$ and Java, the connection between $\mathrm{C}++$ and Java programs is done thanks to JNI (Java Native Interface). The ontology is edited and created with the Protég $\epsilon^{5}$ software. The rules are edited and created with the SWRL6 Plugin. The results were obtained with the Java Constraint Library 7 , and the KAON28 reasoner.

\subsection{Evaluation}

The evaluation is done on a ROI defined by an expert neuroanatomist. It includes the superior frontal gyrus, the middle frontal gyrus, the inferior frontal gyrus, the precentral gyrus, the postcentral gyrus, the central sulcus, the superior precentral sulcus, the intermediate precentral sulcus, the inferior precentral sulcus, the superior frontal sulcus, and the inferior frontal sulcus regions. We compared the results provided by the system to the labels given by the expert considered as a gold-standard. The same procedure was applied to the five MRI datasets except for the matching rules, since orientations were not considered in case of pathological data. For each case, we report the type of the image (normal versus pathological), the number of patches extracted from the selected ROI, the number of consistent interpretations inferred for the patches, the number of user interactions needed to reach the best interpretation for the patches, the accuracy of patch labels (defined as the ratio of the accurately labelled patches over the total number of patches in ROI), and finally the accuracy for the sulci segments (defined as the ratio of the accurately labelled sulci segments over the total number of sulci segments in the ROI).

Table 1 reports the results for the five cases. We observe that the identification of the patches is complete after a reasonable number of interactions with the user (about 3). The sulci identification is quite accurate, although some segments are

Table 1. Results of the experiments

\begin{tabular}{|l|c|c|c|c|c|}
\hline Brain MRI data & $\mathbf{1}$ & $\mathbf{2}$ & $\mathbf{3}$ & $\mathbf{4}$ & $\mathbf{5}$ \\
\hline Normal (N) versus pathological (P) & $N$ & $N$ & $N$ & $P$ & $P$ \\
\hline Nb. of patches in ROI & 16 & 18 & 16 & 17 & 15 \\
\hline Nb. of possible interpretations inferred for patches & 7 & 6 & 6 & 4 & 3 \\
\hline Nb. of user interactions to best interpretation computing & 3 & 3 & 3 & 2 & 2 \\
\hline Accuracy (patches)(\%) & 100 & 100 & 100 & 100 & 100 \\
\hline Accuracy (sulci segments)(\%) & 92.5 & 92.5 & 91 & 94.3 & 90.1 \\
\hline
\end{tabular}

3 http://brainvisa.info/index_f.html

4 http://www.irisa.fr/vista/Themes/Logiciel/VIsTAL/VIsTAL.html

5 http://protege.stanford.edu/

6 http://www.w3.org/Submission/SWRL/

7 http://liawww.epfl.ch/JCL/ (a CSP solver)

8 http://kaon2.semanticweb.org/ (an inference engine for rule-extended ontologies) 


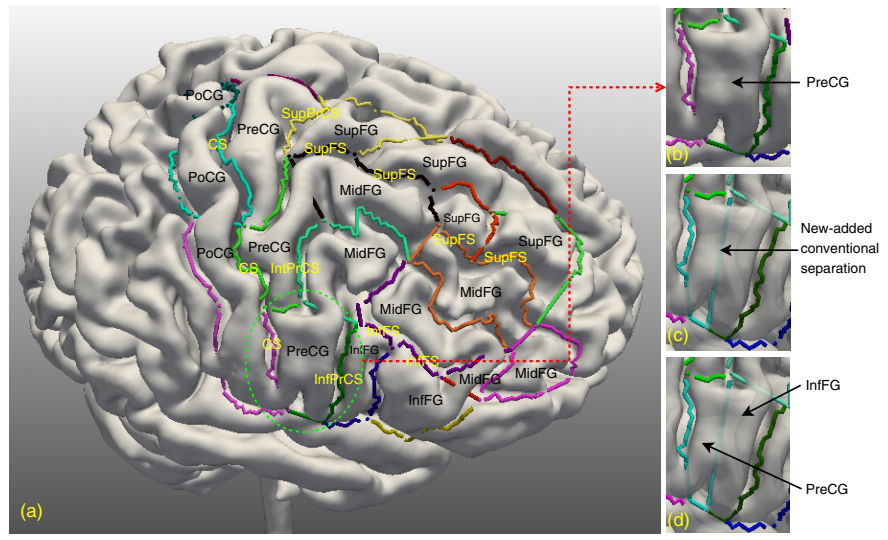

Fig. 4. (a) Inferred labels for the ROI and (b)(c)(d) influence of irrelevant conventional separations

not well classified due to the insufficient precision in the computation of the orientations between the patches and the segments. It is important to notice that the system performance is not decreased in pathological cases in spite of significant structures displacement according to the expert. An example of inferred annotations is reported figure 4 a. We observe on figure 4 (b,c,d) that if we add conventional separations where it is not necessary, then the system becomes less accurate. Regarding the time computing, the Atlas-based pre-labelling of the patches, the CSP reasoning, and the KAON2 reasoning take about 1 minute.

\section{Conclusion}

We have presented a hybrid system developed to semi-automatically label brain MRI images with semantic annotations and the first results obtained so far. The presented approach is novel with respect to several aspects: (1) the use of a CSP solver to select consistent interpretations of the gyri, (2) the easy generation of semantically-rich annotations of gyral/sulcal structures, (3) the use of explicit prior knowledge described in a formal ontology, (4) its represention in OWL, the Ontology Web Language, to facilitate knowledge sharing. Our first experiments using both normal and pathological data are very promising. We plan to still improve the system in the future with respect to several aspects. The definition of the conventional separations is defined manually in the current implementation, and could be automated. The use of atlases as a means to produce the initial hypotheses could be optimized, e.g. by using different types of numerical atlases, more adapted to the particular case under study. The orientations' computation could be improved, e.g. based on [13. Finally, the ontology could be refined, in order to include more fine-grained gyri and sulci, and could be directly used to derive the orientations' definitions in the CSP problem. Moreover, the dependence of the system performance on initial processing steps (especially the definition of conventional separations) should be further investigated. 
Acknowledgement. We thank Louis Collins from the Montreal Neurological Institute for providing us with the SPAMs database, Christian Barillot for his revision of the manuscript, and the Regional Council of Brittany for supporting this project.

\section{References}

1. Ruttenberg, A., Clark, T., Bug, W., Samwald, M., Bodenreider, O., Chen, H., Doherty, D., Forsberg, K., Gao, Y., Kashyap, V., Kinoshita, J., Luciano, J., Marshall, M., Ogbuji, C., Rees, J., Stephens, S., Wong, G., Wu, E., Zaccagnini, D., Hongsermeier, T., Neumann, E., Herman, I., Cheung, K.: Advancing translational research with the Semantic Web. BMC Bioinformatics 8(Suppl. 3), S2 (2007)

2. Corcho, O.: Ontology based document annotation: trends and open research problems. Int. Journal of metadata, semantics and ontologies 1(1), 47-57 (2006)

3. Jannin, P., Morandi, X., Fleig, O., Le Rumeur, E., Toulouse, P., Gibaud, B., Scarabin, J.: Integration of sulcal and functional information for multimodal neuronavigation. Journal of Neurosurgery 96(4), 713-723 (2002)

4. Tzourio-Mazoyer, N., Landeau, B., Papathanassiou, D., Crivello, F., Etard, O., Delcroix, N., Mazoyer, B., Joliot, M.: Automated anatomical labeling of activations in SPM using a macroscopic anatomical parcellation of the MNI MRI Single-Subject brain. Neuroimage 15(1), 273-289 (2002)

5. Cachia, A., Mangin, J., Rivière, D., Papadopoulos-Orfanos, D., Kherif, F., Bloch, I., Régis, J.A.: A generic framework for parcellation of the cortical surface into gyri using geodesic Voronoï diagrams. Medical Image Analysis 7(4), 403-416 (2003)

6. Fischl, B., van der Kouwe, A., Destrieux, C., Halgren, E., Ségonne, F., Salat, D., Busa, E., Seidman, L., Goldstein, J., Kennedy, D., Caviness, V., Makris, N., Rosen, B., Dale, A.: Automatically parcellating the human cerebral cortex. Cerebral Cortex 14, 11-22 (2004)

7. Clouchoux, C., Coulon, O., Anton, J., Mangin, J., Régis, J.: A new cortical surface parcellation model and its automatic implementation. In: Larsen, R., Nielsen, M., Sporring, J. (eds.) MICCAI 2006. LNCS, vol. 4191, pp. 193-200. Springer, Heidelberg (2006)

8. Franz, B., Diego, C., Deborah, L., Daniele, N., Peter, F.P.S.: The Description Logic Handbook: Theory, Implementation, and Applications. Cambridge University Press, Cambridge (2003)

9. Le Goualher, G., Barillot, C., Bizais, Y.: Modeling cortical sulci with active ribbons. IJPRAI 11(8), 1295-1315 (1997)

10. Collins, D.L., Zijdenbos, A.P., Baaré, W.F.C., Evans, A.C.: INSECT: Improved cortical structure segmentation. In: Kuba, A., Sámal, M., Todd-Pokropek, A. (eds.) IPMI 1999. LNCS, vol. 1613, pp. 210-223. Springer, Heidelberg (1999)

11. Krzysztof, A.: Principles of Constraint Programming (2003)

12. Ono, M., Kubik, S., Abernathey, C.: Atlas of the Cerebral Sulci (1990)

13. Yann, H., Aline, D.: Qualitative spatial relationships for image interpretation by using semantic graph. In: Escolano, F., Vento, M. (eds.) GbRPR 2007. LNCS, vol. 4538, pp. 240-250. Springer, Heidelberg (2007)

14. Mechouche, A., Golbreich, C., Gibaud, B.: Towards a hybrid system using an ontology enriched by rules for the semantic annotation of brain MRI images. In: Marchiori, M., Pan, J.Z., de Marie, C.S. (eds.) RR 2007. LNCS, vol. 4524, pp. 219-228. Springer, Heidelberg (2007) 